\title{
Spin-charge separation in quasi one-dimensional organic conductors
}

Published online: 26 June 2003

(C) Springer-Verlag 2003

\begin{abstract}
Matter is excited by adding an electron or extracting one. These excitations can move in the bulk material almost like a free particle, carrying an electronic charge and spin. The electrons try to avoid each other by Coulomb repulsion and also interact magnetically. If they are confined to one dimension, charge and spin excitations are separated and move independently due to the strong interaction. The unique properties of one-dimensional systems are revealed in a number of experiments on strongly anisotropic materials. Here we review the theoretical models and the experimental indications for the unusual behavior of quasi one-dimensional organic conductors.
\end{abstract}

\section{Introduction}

Since our world is three-dimensional, there seems to be no good reason to think about lower or higher dimensions; with a few exceptions, when it turns out to be mathematically advantageous to treat a problem in higherdimensional space. In the opposite case of lower dimensions, it is more obvious that things are simpler and problems are more easily solved. For example, dropping a ball is a one-dimensional problem with the acceleration directed to the ground. Also, external constraints can restrict the motion to one or two dimensions: balls on a billiard table allow us to study the collision and momentum transfer in two dimensions; cars on a rail are the common model for a one-dimensional impact. In all of these cases, however, the physics remains the same, only the description got simpler due to the reduced number of dimensions.

The situation becomes different when the spatial extension is so severely confined that quantum effects

\footnotetext{
M. Dressel ( $)$

1. Physikalisches Institut, Universität Stuttgart,

Pfaffenwaldring 57, 70550 Stuttgart, Germany

e-mail: dressel@pi1.physik.uni-stuttgart.de

Tel.: +49-711-6854946
}

Fax: +49-711-6854886 become important. The basic notion of quantum mechanics is that particles can also be described by waves. The energy $E$ of the particle is related to the frequency $\omega$ by $E=\hbar \omega$, and the momentum $p$ is related to the wavelength $\lambda$ by $p=\hbar k=h / \lambda$, where $\hbar=h / 2 \pi$ is Planck's constant and $k$ is the wave vector. Roughly speaking, quantum behavior becomes appreciable if the geometry is comparable to the wavelength of the particles, which is a few Angström. The confinement of electrons to two dimensions is achieved, for example, at the interface between two semiconductors of different doping. Besides the considerable technological applications of these two-dimensional electron gases in micro-electronics, there are a number of phenomena which require the restricted geometry, most prominent being the quantum Hall effect. Going even further, oneand zero-dimensional structures can be obtained by a lateral confinement of a two-dimensional electron gas, the so-called quantum wires and quantum dots.

Again, the physics in one dimension is fundamentally different from the two- and three-dimensional cases. The well-known Fermi-liquid theory to describe the interacting electron gas breaks down in one dimension and the Luttinger liquid takes its place. The implications are certain power laws in the energy dependence of various physical quantities and a separation of the spin and charge propagation. While the theoretical predictions have been around for decades, it is only recently that experimental evidence has been collected which reveals this behavior.

\section{Organic conductors}

There are presently two different experimental approaches to the physics of one-dimensional conductors; one is fabricating mono-atomic linear structures on semiconductor surfaces, while the other makes use of strongly anisotropic crystals. In the last three decades a joint effort of chemists, materials scientists and physicists has succeeded in synthesizing crystals which exhibit a metallic conductivity along one axis, but are more or less insulating in the perpendicular directions. Note that 
these materials grow as single crystals of macroscopic size, typically a few millimeters; they often have a needle-like shape. Although there are a number of inorganic one-dimensional metals, such as $\mathrm{K}_{2}\left[\mathrm{Pt}(\mathrm{CN})_{4}\right.$ $\left.\mathrm{Br}_{0.3}\right] \cdot 3 \mathrm{H}_{2} \mathrm{O}$, which is better known as $\mathrm{KCP}, \mathrm{NbSe}_{3}$, or the blue bronze $\mathrm{K}_{0.3} \mathrm{MoO}_{3}$ (Monceau 1985), organic compounds turn out to be more suitable for exploring the phenomena of one-dimensional conductors.

The breakthrough of these synthetic metals happened in the 1970s with TTF-TCNQ and the TMTSF salts which show a metallic behavior down to low temperatures. In general the compounds consist of stacks of planar molecules with the atomic orbitals overlapping along the stack. In the perpendicular directions the conductivity is orders of magnitude lower because the distance between the stacks is large. There are two prerequisites for a good electronic transport: the overlap of the orbitals and an electronic charge transfer between donor and acceptor molecules to generate 'half-filled' bands. Tetrathiofulvalene-tetracyanoquinomethane (TTF-TCNQ) is a charge-transfer compound with separate stacks of the cations TTF (donors) and anions TCNQ (acceptors). By synthesizing tetramethyl-tetraselenafulvalene (TMTSF), an enlarged variant of TTF, and by replacing TCNQ by $\mathrm{PF}_{6}^{-}$, in 1979 Klaus Bechgaard and Denis Jérome (Jérome et al. 1980) successfully produced the first organic superconductor (Jérome and Schulz 1982).

The planar organic TMTSF molecules stack along the $a$-direction with an average distance of $3.645 \AA$, which is less than twice the van der Waals radius of Se necessary for the orbital overlap. In the $b$-direction the coupling between the chains is small, while in the third direction the stacks are separated by the inorganic anion, like $\mathrm{PF}_{6}^{-}$, $\mathrm{AsF}_{6}^{-}, \mathrm{ClO}_{4}^{-}, \mathrm{Br}^{-}$, etc., as depicted in Fig. 1. Each organic molecule transfers half an electron to the anions. In general a small dimerization leads to pairs of organic molecules, resulting in a half-filled band. In addition, spontaneous charge disproportionation, called charge ordering (CO), may divide the molecules into two nonequivalent species. Due to the instability of the quasi one-dimensional Fermi surface, at ambient pressure (TMTSF) ${ }_{2} \mathrm{PF}_{6}$ undergoes a transition to a spin-densitywave (SDW) ground state at $T_{\mathrm{SDW}}=12 \mathrm{~K}$. Applying pressure or replacing the $\mathrm{PF}_{6}^{-}$anions by $\mathrm{ClO}_{4}^{-}$leads to a stronger coupling in the second direction: i.e., the material is more two-dimensional (Farges 1994; Ishiguro et al. 1998).

During recent years the family of TMTCF salts (where $C$ is one of the chalcogenes selenium or sulfur) was intensively explored and became the model system of quasi one-dimensional conductors. By external pressure or substitution of anions (chemical pressure) the interchain coupling increases and thus the dimensionality crosses over from a strictly one-dimensional to a more two- or three-dimensional system. Over the past two decades various groups have contributed to the rich phase diagram as displayed in Fig. 2. As well as the Mott insulating state, spin Peierls, antiferromagnetic insulator,

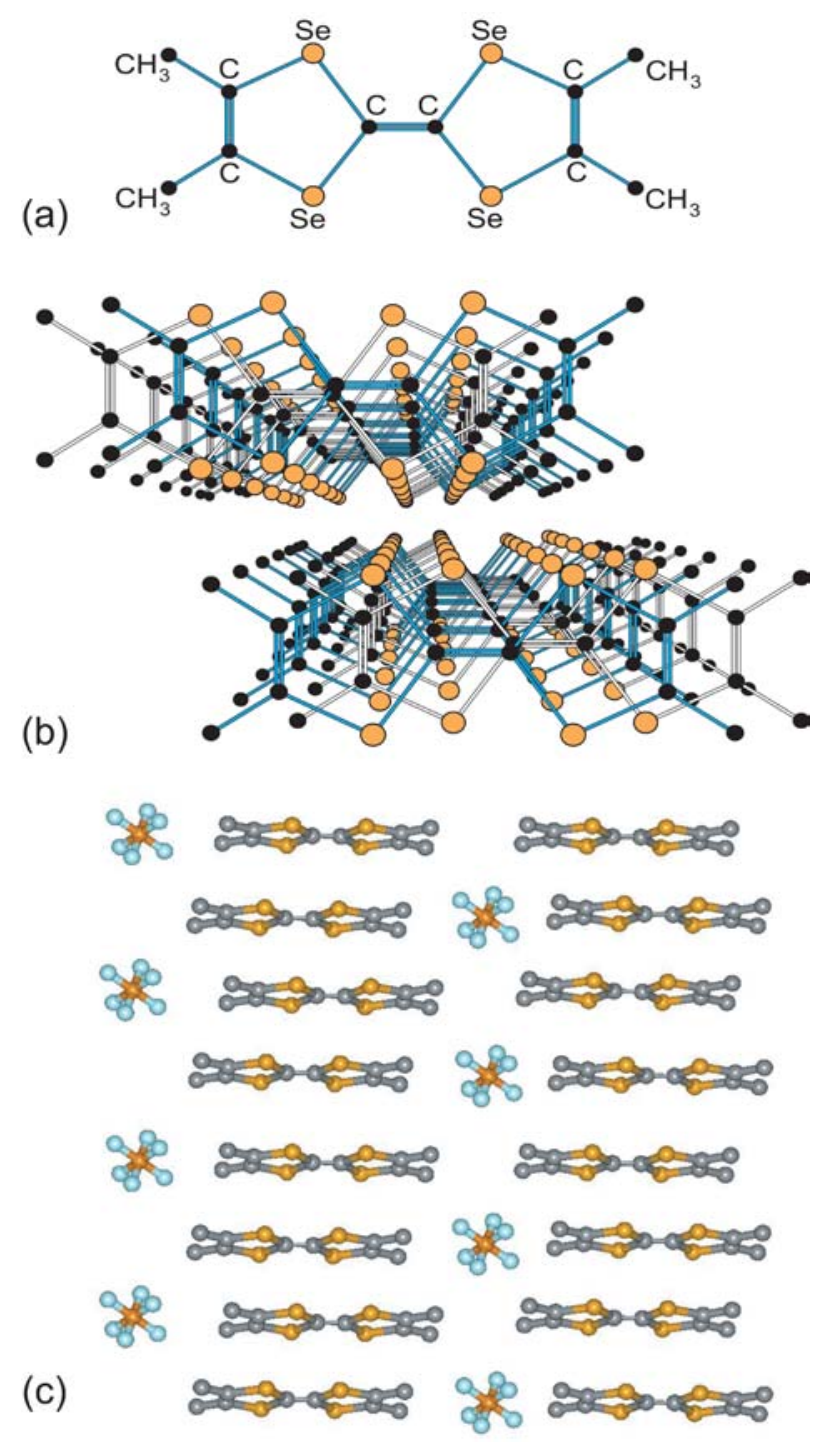

Fig. 1 a TMTSF molecule; b view along the stacks of TMTSF ( $a$ direction), and $\mathbf{c}$ perpendicular to them (b-direction). Along the $c$ direction the stacks of the organic molecules are separated by the $\mathrm{PF}_{6}^{-}$anions

spin-density-wave, and superconductivity which occur when the coupling between the stacks is increased, in addition the metallic state changes its behavior, going from a Luttinger liquid to a Fermi liquid. This can be nicely seen in electronic properties like the $c$-axis resistivity (Moser et al. 1998) or the optical conductivity (Dressel et al. 1996); spin dynamics, on the other hand, does not show any fundamental change going from TMTTF to TMTSF except that the spins are less localized (Dumm et al. 2000). Most recently thermal transport also indicated that the charge degrees of freedom behave in a totally different way from the spin degrees of freedom (Lorenz et al. 2002). 


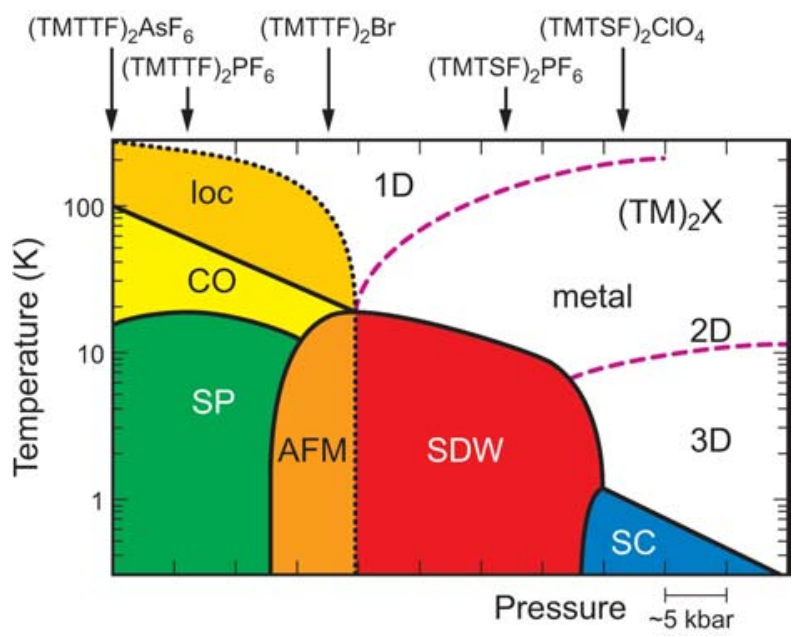

Fig. 2 The phase diagram of the quasi one-dimensional TMTTF and TMTSF salts as established by Jérome and coworkers (Jérome 1991). For the different compounds the ambient-pressure position in the phase diagram is indicated. Going from left to right, the materials get less one-dimensional due to the increasing interaction in the second and third direction. Here loc stands for charge localization, $C O$ for charge ordering, $S P$ for spin-Peierls, $A F M$ for antiferromagnet, $S D W$ for spin density wave, and $S C$ for superconductor. The description of the metallic state changes from a onedimensional Luttinger liquid to a two and three-dimensional Fermi liquid. While some of the boundaries are clear phase transitions, the ones indicated by dashed lines are better characterized as a crossover

\section{Fermi liquid}

In conventional metals, such as aluminum, copper or gold, the conduction electrons travel independently of each other. The optical response of metals was described by Paul Drude in 1900. The frequency-dependent conductivity $\sigma(\omega)$ is determined by a relaxation time $\tau$, and the spectral weight $\int \sigma(\omega) \mathrm{d} \omega=\pi N e^{2} / 2 m$ which measures the density of conduction electrons $N$ and the carrier mass $m$; here $e$ denotes the electronic charge and $\omega$ the frequency (Dressel and Grüner 2002).

Soon it was realized that the electrons do not move freely through the crystal but interact with the underlying lattice; hence the free-electron mass is replaced by the bandmass. A similar renormalization can account for the Coulomb interaction between the electrons which is of minor importance in simple metals. However, in some intermetallic compounds containing $\mathrm{Ce}$ or $\mathrm{U}$, for example, it becomes dominant at low temperatures; since the effective mass is enhanced by a few hundred times the bandmass; these materials are called heavy-fermion systems. In all cases the electron-phonon or electronelectron interactions lead to a renormalization of the quasi-particles, but leave their character unchanged. The electrons still obey Fermi statistics and this type of electron liquid is called interacting Fermi liquid (Pines and Nozières 1966). As pointed out by Lev Landau nearly half a century ago (Landau 1957), the low-energy excitations of a system with interactions can be mapped
1:1 onto a non-interacting system using proper renormalization. The bare electrons can be pictured as being 'dressed' with a cloud of virtual electron-hole pair excitations, but the quasi-particles still carry the same charge and spin as free electrons. This means that the electronic charge and spin propagate coherently in an interacting Fermi liquid, just as they do in a free-electron gas. Since the Fermi energy $E_{F}$ corresponds to approximately $10^{4} \mathrm{~K}$ and at normal temperatures only a small fraction of particles $\left(T / T_{F}\right)$ participate in the scattering processes, the quasi-particles are robust against small displacements away from the Fermi surface with a lifetime diverging as $\tau \propto\left(E-E_{F}\right)^{-2}$. This leads to the well-known $T^{2}$ dependence of $\rho(T)$ and $\sigma(\omega) \propto \omega^{-2}$ (Dressel and Grüner 2002).

Under certain circumstances, notably when the electron system is driven close to an instability, or when the electronic structure is highly anisotropic, the renormalized Fermi-liquid picture is not valid any more; other types of quantum liquids may replace it, which are often described as non-Fermi liquids.

\section{Luttinger liquid}

Partially based on the work of Tomonaga (1950), in 1963 M. Luttinger suggested a simple, exactly solvable model to describe one-dimensional metals (Luttinger 1963). A single parameter $K$ is used to account for all physical quantities (at least close to the Fermi edge): $1-K$ measures the strength and sign of the interaction. The band structure is approximated by two branches at the Fermi wave vector $\pm k_{F}$ (see Fig. 3) and the Coulomb interaction is considered as long as the momentum exchange is small and the number of particles is conserved in each branch. During recent years other one-dimensional models, such as the Hubbard model, and their properties at low energies could be characterized as Luttinger liquids. As suggested by Haldene (1981), their formalism is now used to describe the universal lowenergy phenomenology of gapless one-dimensional quantum systems. It is characterized by (1) the absence of quasi-particles carrying electron quantum numbers in the

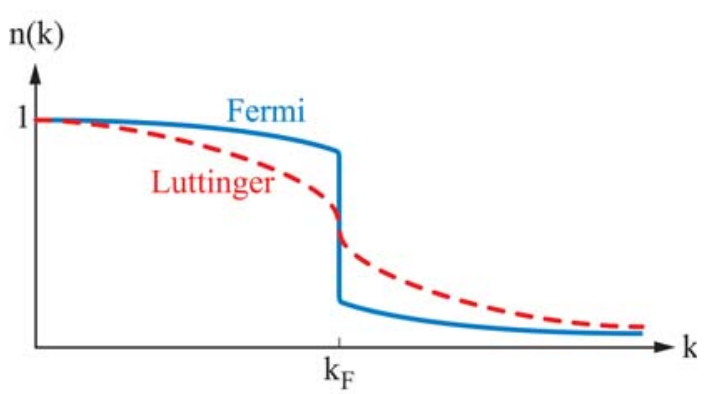

Fig. 3 The momentum distribution function of a Fermi liquid shows a step at the Fermi wave vector $k_{F}$ for $T=0$ even for finite interactions. In the case of a Luttiger liquid, a power-law behavior is predicted 


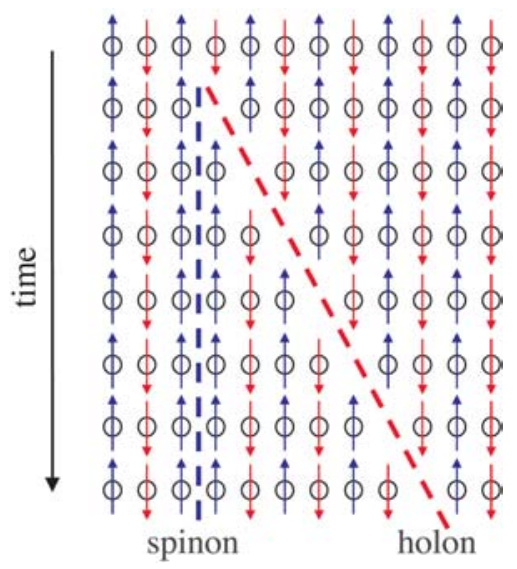

Fig. 4 The time evolution of spin and charge excitations in a onedimensional system shows the decay of an excitation into a chargecarrying holon and a spin-carrying spinon. Going from the first to the second row, an electron is extracted. By hopping of neighboring electrons, the hole moves to the right as time elapses while the excitation of two parallel spins next to each other remains in place

vicinity of the Fermi-surface; (2) spin-charge separation; and (3) anomalous dimensions of fermions which produce non-universal power-law decay of correlation functions (Voit 1995).

Fundamental paradigms of solid-state physics are no longer valid in one dimension. As sketched out in Fig. 4, spin and charge can propagate independently and with different velocities in the form of collective excitation modes called spinons (neutral spin excitations) and holons (spin-less charge excitations). The differences between Fermi and Luttinger liquids are best visualized by the momentum distribution function displayed in Fig. 4. While a Fermi liquid exhibits a finite step of $n(k)$ at $k_{F}$ at $T=0$, the Luttinger liquid varies as $\left(k-k_{F}\right)^{\alpha}$ where $\alpha=\left(K+K^{-1}-2\right) / 4$. The power-law behavior observed in various physical quantities has a consequence that the electrons decay in collective excitations. The one-electron distribution function, for example, is given by $N(E) \propto\left(E-E_{F}\right)^{\alpha}$. The NMR relaxation rate shows a $\mathrm{T}_{1}^{-1}$ $(T) \propto T^{K}$ behavior and similar laws can be found for thermodynamic quantities like the susceptibility and specific heat. For a quarter-filled system, as is the case for the Bechgaard salts, the temperature-dependent resistivity should exhibit a $\rho(T) \propto T^{16 K-3}$ behavior. For the frequency-dependent conductivity $\sigma(\omega) \propto \omega^{16 K-5}$ is predicted (Giamarchi 1991, 1997; Giamarchi and Millis 1992)

The verification of the Luttinger model in real systems remains a challenge, because the predictions were deduced for the extreme case of low energies $(T 0, \omega 0)$ and strictly one dimension. Both assumptions can be approximated experimentally only to a certain degree. During recent years a large amount of evidence has been collected on the quasi one-dimensional organic conductors, which by now allows us to draw a more or less consistent picture.

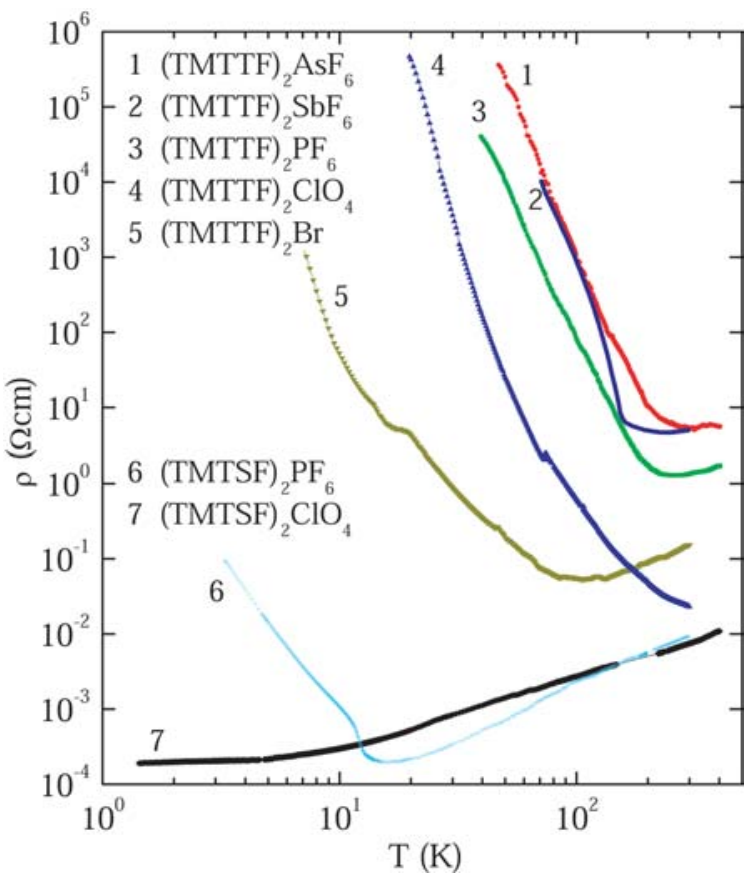

Fig. 5 Temperature dependence of the electrical resistivity along the stacks of various TMTSF and TMTTF salts with different anions as indicated (Dressel et al. 2001)

\section{Charge degree of freedom}

The charge dynamics of a metal can be studied by the temperature- and frequency-dependent transport. Figure 5 displays the temperature dependence of the dc resistivity of different TMTCF compounds measured along the stacks. The TMTTF salts show a flat or weakly metallic behavior above 100-200 K. The strong electron-electron correlations in these one-dimensional systems induce a gap in the electronic excitations (Mott-Hubbard gap); thus at low temperatures the transport is activated with an energy which decreases when going from (TMTTF) ${ }_{2} \mathrm{AsF}_{6}$ to (TMTTF $)_{2} \mathrm{Br}$. The (TMTSF) $)_{2} X$ salts, on the other side of the phase diagram (see Fig. 2), show a metallic behavior of the resisitivity down to very low temperatures with $\rho(T) \propto T$, which gives $K \approx 0.25$. At $T_{\mathrm{SDW}}=12 \mathrm{~K}$, (TMTSF) ${ }_{2} \mathrm{PF}_{6}$ undergoes a SDW transition to an insulator.

The crossover from a Mott insulator to an unconventional metal can also be observed in the electrodynamic properties. At room temperatures or at high frequencies the optical conductivity of different TMTCF salts is very similar. As seen in Fig. 6, the low-temperature properties $(T \approx 20 \mathrm{~K})$ exhibit an energy gap of a few hundred wavenumbers. The low-frequency spectra (below $10 \mathrm{~cm}^{-1}$ ) reflect the completely different transport behavior: in (TMTSF $)_{2} \mathrm{PF}_{6}$ and (TMTSF) $)_{2} \mathrm{ClO}_{4}$ a very narrow contribution of quasi-free electrons is present which is responsible for the dc conductivity. This behavior is explained by the increasing interchain coupling as we go to the right side of the phase diagram of Fig. 2. 


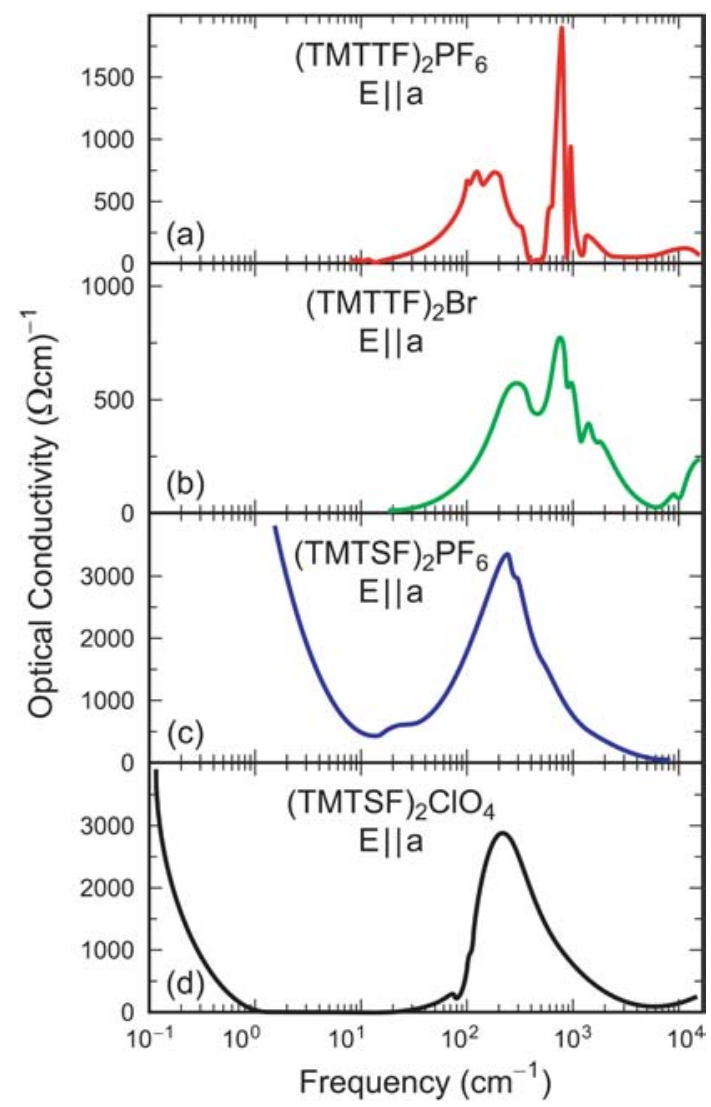

Fig. 6 On-chain optical conductivity of different (TMTCF $)_{2} X$ compounds at $T \approx 20 \mathrm{~K}$ (Dressel et al. 1996; Schwartz et al. 1998; Vescoli et al. 1998). Note the logarithmic frequency scale

The extremely small spectral weight agrees with the reduced density of states observed by photoemission experiments (Dardel et al. 1993); instead of a stepfunction as for conventional metals, a power-law is found. The fingerprint of a quasi one-dimensional metal is the correlation-induced gap around $200 \mathrm{~cm}^{-1}$, above which the optical conductivity of all TMTSF salts follows a power law $\sigma(\omega) \propto \omega^{-1.3}$, corresponding to $K \approx 0.23$ (Schwartz et al. 1998). The deviations of the NMR relaxation rate from the Korringa law yield an even smaller value of $K$ (Wzietek et al. 1993), all indicating strong and long-range interactions.

\section{Spin degree of freedom}

In order to investigate the spin degrees of freedom, detailed studies of the electron spin resonance (ESR) were performed on most of the TMTCF salts (Dumm et al. 2000). The integrated absorption is proportional to the spin susceptibility; at high temperatures $\chi(T)$ of the (TMTTF) ${ }_{2} X$-compounds (Fig. 7) correspond to a spin $1 / 2$ antiferromagnetic Heisenberg chain with exchange constants $J=420-500 \mathrm{~K}$. Although TMTSF salts are metallic down to low temperatures, for $T>100 \mathrm{~K}$ the temperature dependence of $\chi(T)$ can be described within the frame-

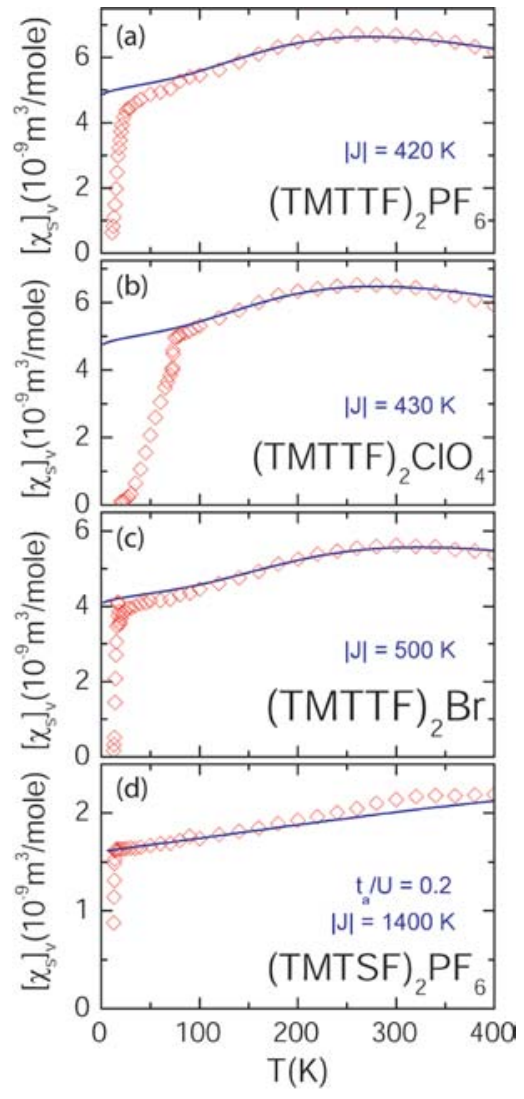

Fig. 7 Temperature dependence of the spin susceptibility $\left(\chi_{s}\right)_{v}$ at constant volume of different TMTCF-salts as obtained by ESR intensity. The lines in $\mathbf{a}, \mathbf{b}$ and $\mathbf{c}$ correspond to a $S=1 / 2$ AFM Heisenberg chain with $J=420 \mathrm{~K}, J=430 \mathrm{~K}$, and $J=500 \mathrm{~K}$, the line in the lowest frame (d) corresponds to a fit by an advanced model with $t_{a} / U=0.2$ (Dumm et al. 2000)

work of the Hubbard model in the limit of strong Coulomb repulsion with $J \approx 1,400 \mathrm{~K}$ and $t_{a} / U=0.2$.

A comparison of Figs. 5 and 7 clearly shows that going from the strictly insulating (TMTTF) ${ }_{2} \mathrm{PF}_{6}$ to the highly metallic (TMTSF) ${ }_{2} \mathrm{ClO}_{4}$ there is a sudden change in the charge-transport properties when the transfer integral becomes comparable to the charge gap, while the spin dynamics varies continuously as described by a steadily increasing exchange constant. The resistivity in (TMT$\mathrm{TF})_{2} X$ strongly increases below $100 \mathrm{~K}$; in contrast the susceptibility and also the line width vary only slightly. This behavior indicates the separation of spin and charge degrees of freedom.

\section{Spin and charge ordering}

In the TMTTF salts, spin and charge are localized onto the organic molecules in the stacks leading to an electrical insulating state and magnetic behavior of a Heisenberg chain. While at elevated temperatures the distribution is homogeneous, modulations of the charge and of the spin distributions are observed as the temperature is lowered. 


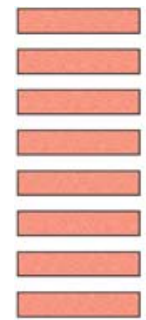

(a)

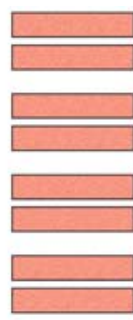

(b)

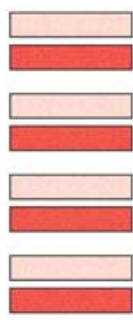

(c)

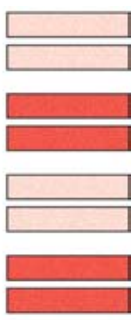

(d)
Fig. 8 a Stack of equally spaced organic molecules with charge $\rho=\rho_{0}=1 / 2 e$ per molecule; $\mathbf{b}$ the dimerization of the stack leads to alternating distances. Charge ordering modulates the electronic charge $\rho$ per molecule to $\rho_{0}+\delta$ and $\rho_{0}-\delta$; as indicated by the gray values; as well as a ' -+-+ ' pattern (c), a '- - + + pattern is also possible (d)

A possible mechanism of charge ordering is the localization of electrons on a lattice due to long-range Coulomb repulsion, which is the lattice version of the Wigner crystallization (Fig. 8). Even in the charge-ordered state, the spin degrees of freedom are still active. Clear evidence of the charge-order phase transition around $T_{\mathrm{CO}} \approx 60-100 \mathrm{~K}$ was given by the measurements of the dielectric response which shows a ferroelectric behavior (Monceau et al. 2001), and by NMR spectroscopy, where sites of molecules with charge $\rho=\rho_{0}+\delta$ and $\rho_{0}-\delta$ have been identified with a charge disproportion as large as $\delta=1 / 4$ (Chow et al. 2000; Zamborszky et al. 2002).

At much lower temperatures, the TMTCF salts can undergo an additional phase transition, which results in a variety of magnetically ordered ground states such as spin-Peierls, antiferromagnetic and spin density wave state (Fig. 2). In the case of a spin-Peierls transition, the formation of spin pairs leads to a small modulation of the underlying lattice; the non-magnetic ground state is identified by a drop of the susceptibility below $T_{\mathrm{SP}}=19 \mathrm{~K}$ as seen in Fig. 7a. The spin-density-wave is defined as an antiferromagnetic ground state of a metal with a spatially periodic arrangement of the electron spin. Below $T_{\mathrm{SDW}}=12 \mathrm{~K}$ the electron gas becomes instable due to nesting of the Fermi surface, which results in a collectively ordered state of an itinerant antiferromagnet. Due to the superstructure, a gap in the electronic density of states at $k_{F}$ opens and therefore the spin susceptibility vanishes rapidly (Fig. 7d). Recent calculations (Shibata et al. 2001; Clay et al. 2003) indicate that the two possible charge-ordering patterns sketched out in Fig. 8c, d are consistent with the spin-Peierls and spin-density-wave state which subsequently occurs at much lower temperatures. The different energy scales of both ordering phenomena and the distinct response upon applied pressure indicates that the spin and charge degrees of freedom behave differently in these quasi one-dimensional systems.
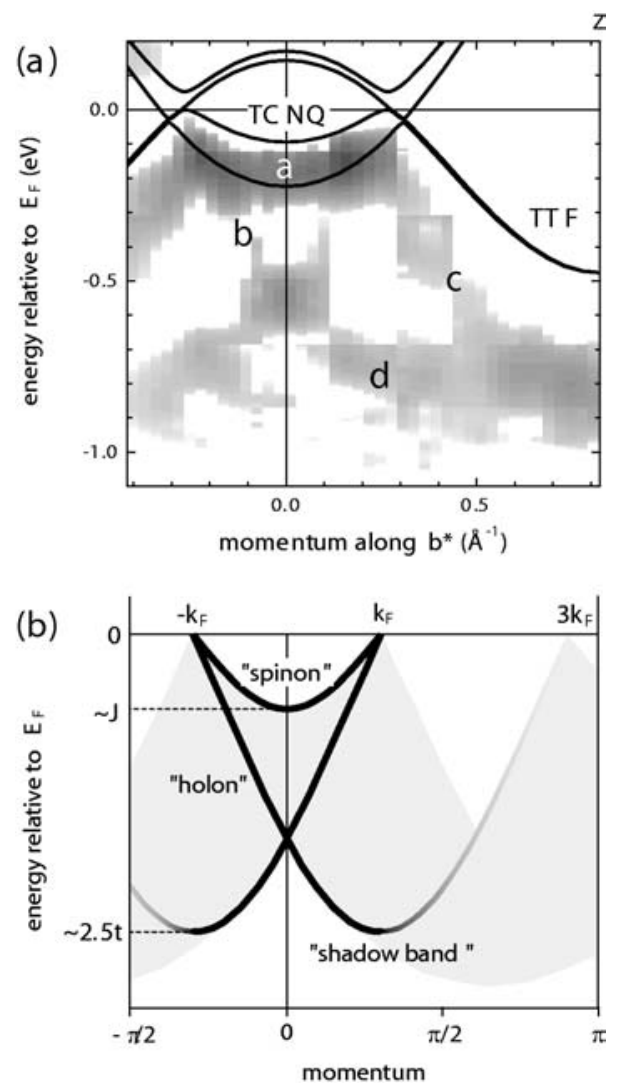

Fig. 9 a Gray-scale plot of the ARPES dispersion and the conduction bands obtained from density functional band theory of TTF-TCNQ. b Schematic electron removal spectrum of the onedimensional Hubbard model at finite doping (Claessen et al. 2002)

\section{Spin-charge separation}

In one dimension the electron-electron interaction is so strong that electron-hole excitations delocalize the electron until it is totally incoherent. What remains are collective spin and charge excitations which behave independently and lead to a spatial separation of spin and charge. In a gedanken experiment, an extra hole in a onedimensional metal would decay into a holon and a spinon as depicted in Fig. 4. The spectral function, which also probes the propagation velocities for charge and spin fluctuations, is expected to show two distinct features with a different momentum dependence, non-universal power-law singularities (depending on $K$ ), and with a shift of spectral weight to higher energies.

Figure 9a displays the dispersion of the angle-resolved photoemission spectra (ARPES) of TTF-TCNQ, which qualitatively agrees with density functional band calculations. The two pairs of nearly degenerate bands are attributed to the TCNQ chains ( $a$ and $b$ ) and TTF chains (c). According to the one-dimensional Hubbard model, the hole generated by the removal of an electron decays into two collective excitations (holon and spinon). As a consequence the spectrum plotted in Fig. 9b consists of a broad continuum determined by the phase space available 


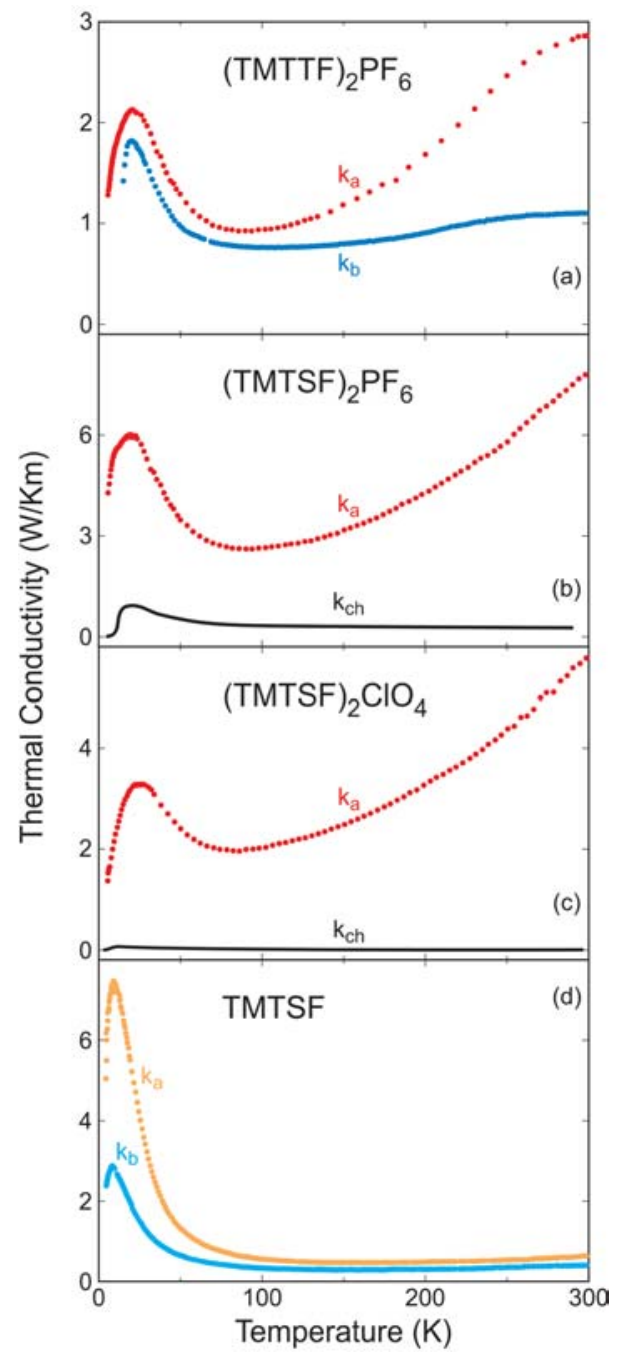

Fig. 10 Temperature dependence of the thermal conductivity along the chain direction for three salts which have very different electrical properties: a $(\mathrm{TMTTF})_{2} \mathrm{PF}_{6}$, which is an insulator, b (TMTSF $)_{2} \mathrm{PF}_{6}$, which is a metal until it turns into a SDW insulator at $12 \mathrm{~K}$, and $\mathbf{c}$ (TMTSF) ${ }_{2} \mathrm{ClO}_{4}$, which remains metallic down to the superconducting transition around $1 \mathrm{~K}$; d the neutral molecular crystal TMTSF only exhibits the lattice contribution (Lorenz et al. 2002). The curves $k_{a}$ correspond to the thermal conductivity along the stacking direction, while $k_{b}$ is measured perpendicular to it; $k_{\mathrm{ch}}$ is the contribution of the charge excitations calculated from the electrical conductivity $\sigma(T)$

for spinon-holon decomposition. In addition, dispersive singularities appear from hole fractionalization into a spinon $(a)$ and a holon $(d)$ (Claessen et al. 2002).

Most recently, experiments on the heat transport in the quasi one-dimensional Bechgaard salts have found that the spin and charge excitations have distinctly different thermal conductivities (Lorenz et al. 2002). Whereas the electrical current probes the charge response only, the heat current is sensitive to charge and spin excitations. As shown in Fig. 10, the temperature dependence of the thermal conductivity $k_{a}(T)$ displays a similar behavior, although their electrical properties are very much different.
Acoustic phonons are responsible for the low-temperature peak (around $20 \mathrm{~K}$ ) in Fig. 10. They are the same for all TMTCF salts, are also seen in the perpendicular direction $k_{b}$ and do not change significantly in the neutral molecular crystal TMTSF. The pronounced increase in the thermal conductivity $k_{a}(T)$ at high temperatures $(T>100 \mathrm{~K})$ is due to magnetic or spin excitations. In accordance with ESR data, these are basically the same far all compounds. The surprising discovery is that the electronic contribution to the thermal conductivity $k_{c h}(T)$ is negligible even for the conducting TMTSF compounds. Usually the heat transport by charge excitations dominates the thermal conductivity of a metal, as described by the Wiedemann-Franz law $k_{\mathrm{ch}}(T)=L_{0} T \sigma(T)$ where $L_{0}$ is the Lorenz number. Our estimate reveals that this contribution does not play an appreciable role. This suggests that also in the metallic system the heat transport is dominated (besides phonons) by magnetic excitations, whereas the electrical conductivity is determined by charge excitations. This is a clear evidence for spin-charge separation and thus for non-Fermi-liquid behavior and the concept of a Luttinger liquid in one-dimensional conductors.

\section{Conclusion}

Like the pieces of a puzzle, the various findings on the quasi one-dimensional organic conductors fit together quite well and form a consistent picture. The low-energy properties observed in the frequency and temperaturedependent transport can be described by power-laws with a single exponent. Photoemission experiments do not see a sharp Fermi edge but a reduced spectral weight with an exponential decay; in the dispersion spinon and holon excitation can be distinguished. The occurrence of different temperature dependences for charge transport, in contrast to a rather uniform heat transport due to spin excitations, provides clear evidence of spin-charge separation and the breakdown of the quasi-particle concept. Indeed these organic materials turn out to be a fascinating arena for the study of electronic correlations in one dimension. Besides this qualitative agreement, a detailed theory for the quantitative understanding has to be worked out.

The next step will be to increase the coupling between the chains in a controlled way and to study how the behavior is be modified. This corresponds to a crossover from one to two and eventually three dimensions. By now there is no continuous interpolation between a Luttinger liquid and a Fermi liquid. The theoretical description of this dimensional crossover remains a challenge for future years.

Acknowledgements During the past few years, we have enjoyed collaborations and discussions with R. Claessen, L. Degiorgi, M. Dumm, A. Freimuth, G. Grüner, and J. Voit. 


\section{References}

Chow DS, Zamborszky F, Alavi B, Tantillo DJ, Baur A, Merlic CA, Brown SE (2000) Charge ordering in the TMTTF family of molecular conductors. Phys Rev Lett 85:1698-1701

Claessen R, Sing M, Schingenschlögl U, Blaha P, Dressel M, Jacobsen CS (2002) Spectroscopic signatures of spin-charge separation in the quasi-one-dimensional organic conductor TTF-TCNQ. Phys Rev Lett 88:096401-1-4

Clay RT, Mazumdar S, Cambell DK (2003) Pattern of charge ordering in quasi-one-dimensional organic charge-transfer solids. Phys Rev B 67:115121-1-9

Dardel B, Malterre D, Grioni M, Weibel P, Baer Y, Voit J, Jérome D (1993) Possible observation of a Luttinger-liquid behaviour from photoemission spectroscopy of one-dimensional organic conductors. Europhys Lett 24:687-692

Dressel M, Grüner G (2002) Electrodynamics of solids. Cambridge University Press, Cambridge

Dressel M, Schwartz A, Grüner G, Degiorgi L (1996) Deviations from Drude response in low-dimensional metals: electrodynamics of the metallic state of (TMTSF) ${ }_{2} \mathrm{PF}_{6}$. Phys Rev Lett 77:398-401

Dressel M, Kirchner S, Hesse P, Untereiner G, Dumm M, Hemberger J, Loidl A, Montgomery L (2001) Spin and charge dynamics in Bechgaard salts. Synth Metals 120:719-720

Dumm M, Loidl A, Fravel BW, Starkey KP, Montgomery L, Dressel M (2000) Electron-spin-resonance studies on the organic linear chain compounds (TMTCF $)_{2} X(C=\mathrm{S}, \mathrm{Se}$ and $\left.X=\mathrm{PF}_{6}, \mathrm{AsF}_{6}, \mathrm{ClO}_{4}, \mathrm{Br}\right)$. Phys Rev B 61:511-520

Farges JP (ed) (1994) Organic conductors. Dekker, New York

Giamarchi $T$ (1991) Umklapp process and resistivity in onedimensional fermion systems. Phys Rev B 33:2905-2913

Giamarchi T (1997) Mott transition in one dimension. Physica B 230-232:975-980

Giamarchi T, Millis AJ (1992) Conductivity of a Luttinger liquid. Phys Rev B 46:9325-9331

Haldene FDM (1981) Luttiner liquid theory of one-dimensional quantum fluids. I. Properties of the Luttinger model and their extension to the general 1D interacting spinless Fermi gas. J Phys C 14:2585-2609

Ishiguro T, Yamaji K Saito G (1998) Organic superconductors, 2nd edn. Springer, Berlin Heidelberg New York

Jérome D (1991) The physics of organic conductors. Science 252:1509-1514

Jérome D, Schulz HJ (1982) Organic conductors and superconductors. Adv Phys 31:299-490
Jérome D, Mazaud A, Ribault M, Bechgaard K (1980) Superconductivity in a Synthetic Organic Conductors (TMTSF) ${ }_{2} \mathrm{PF}_{6} . \mathrm{J}$ Phys Lett 41:L95-98

Landau LD (1957) The theory of a Fermi liquid. Sov Phys JETP 3:920-925

Lorenz T, Hofmann M, Grüninger M, Freimuth A, Uhrig GS, Dumm M, Dressel M (2002) Evidence for spin-charge separation in quasi one-dimensional organic conductors. Nature 418:614-617

Luttinger JM (1963) An exactly solvable model of a many-fermion system. J Math Phys 4:1154-1162

Monceau P (ed) (1985) Electronic properties of inorganic quasione-dimensional compounds, part I/II. Reidel, Dordrecht

Monceau P, Nad FY, Brazovskii S (2001) Ferroelectric MottHubbard phase of organic (TMTTF $)_{2} X$ conductors. Phys Rev Lett 86:4080-4083

Moser J, Gabay M, Auban-Senzier P, Jérome D, Bechgaard K, Fabre JM (1998) Transverse transport in $(\mathrm{TM})_{2} X$ organic conductors: possible evidence for a Luttinger liquid. Eur Phys J B 1:39-46

Pines D, Nozières P (1966) The theory of quantum liquids, vol 1. Addison-Wesley, Reading

Schwartz A, Dressel M, Grüner G, Vescoli V, Degiorgi L, Giarmarchi T (1998) On-chain electrodynamics of metallic (TMTSF) ${ }_{2} X$ salts: observation of Tomonaga-Luttinger liquid response. Phys Rev B 58:1261-1271

Shibata Y, Nishimoto S, Ohta Y (2001) Charge ordering in the onedimensional extended Hubbard model: implications to the TMTTF family of organic conductors. Phys Rev B 64:235107$1-5$

Tomonaga S (1950) Remarks on Bloch's method of sound waves applied to many-fermion problems. Prog Theor Phys Kyoto 5:544-569

Vescoli V, Degiorgi L, Henderson W, Grüner G, Starkey KP, Montgomery LK (1998) Dimensionality-driven insulator-tometal transition in the Bechgaard salts. Science 281:1181-1184

Voit J (1995) One-dimensional Fermi liquids. Rep Prog Phys 58:977-1116

Wzietek P, Creuzet F, Bourbonnais C, Jérome D, Bechgaard K, Batail P (1993) Nuclear relaxation and electronic correlations in quasi-one-dimensional organic conductors. II. Experiments. J Phys I Paris 3:171-200

Zamborszky F, Yu W, Raas W, Brown SE, Alavi B, Merlic CA, Baur A (2002) Competition and coexistence of bond and charge orders in (TMTTF) ${ }_{2} \mathrm{AsF}_{6}$. Phys Rev B 66:081103-081106 